Volume 116 No. 1 2017, 31-43

ISSN: 1311-8080 (printed version); ISSN: 1314-3395 (on-line version)

url: http://www.ijpam.eu

doi: 10.12732 /ijpam.v116i1.3

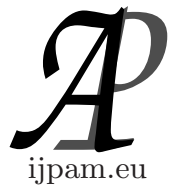

\title{
APPROXIMATE QUADRATIC MAPPINGS IN MODULAR SPACES
}

\author{
Hark-Mahn Kim ${ }^{1}$, Young Soon Hong $2 \S$ \\ ${ }^{1,2}$ Department of Mathematics \\ Chungnam National University
}

99 Daehak-ro, Yuseong-gu, Daejeon, 34134, REPUBLIC OF KOREA

\begin{abstract}
In this article, we investigate an alternative generalized Hyers-Ulam stability theorem of a modified quadratic functional equation in a modular space $X_{\rho}$ using $\Delta_{3}$-condition without the Fatou property on the modular function $\rho$.
\end{abstract}

AMS Subject Classification: 39B82, 39B72, 16W25

Key Words: generalized Hyers-Ulam stability, modular spaces, Fatou property, $\Delta_{3}$-condition

\section{Introduction}

In 1940, S.M. Ulam [12] raised the question concerning the stability of group homomorphisms: Let $G$ be a group and let $G^{\prime}$ be a metric group with the metric $d(\cdot, \cdot)$. Given $\varepsilon>0$, does there exist a $\delta>0$ such that if a mapping $f: G \rightarrow G^{\prime}$ satisfies the inequality

$$
d(f(x y), f(x) f(y))<\delta
$$

for all $x, y \in G$, then there exists a homomorphism

$$
F: G \rightarrow G^{\prime} \text { with } d(f(x), F(x))<\varepsilon,
$$

for all $x \in G$ ? The case of approximately additive mappings was solved by

Received: January 25, 2017

Revised: May 16, 2017

Published: $\quad$ August 29, 2017

$\S_{\text {Correspondence author }}$ (c) 2017 Academic Publications, Ltd. url: www.acadpubl.eu 
D.H. Hyers [5] under the assumption that $X$ and $Y$ are Banach spaces. A generalization of Hyers' theorem was provided by Th.M. Rassias [8] in 1978 and by P. Găvruta [4] in 1994.

The equation $f(x+y)+f(x-y)=2[f(x)+f(y)]$, which may be originated from the important parallelogram equality $\|x+y\|^{2}+\|x-y\|^{2}=2\left[\|x\|^{2}+\|y\|^{2}\right]$ in inner product spaces, is called a quadratic functional equation, and every solution of the quadratic functional equation is said to be a quadratic mapping. The Hyers-Ulam stability problem for the quadratic functional equation was proved by F. Skof [11] for a mapping $f: X \rightarrow Y$, where $X$ is a normed space and $Y$ is a Banach space. S. Czerwik [3] proved the Hyers-Ulam stability of the quadratic functional equation with the sum of powers of norms in the sense of Th. M. Rassias approach using direct method. J.M. Rassias [6] proved the Hyers-Ulam stability of the quadratic functional equation with the product of powers of norms using direct method. On the other hand, C. Borelli and G.L. Forti [2] have proved the generalized Hyers-Ulam stability theorem of the quadratic functional equation whose quadratic difference was controlled by general functions with regularity conditions.

Next, we introduce to recall some basic definitions and remarks of modular spaces with modular functions, which are primitive notions corresponding to norms or metrics, as in the followings.

Definition 1. Let $\chi$ be a linear space.

(a) A function $\rho: \chi \rightarrow[0, \infty]$ is called a modular if for arbitrary $x, y \in \chi$,

(1) $\rho(x)=0$ if and only if $x=0$,

(2) $\rho(\alpha x)=\rho(x)$ for every scalar $\alpha$ with $|\alpha|=1$,

(3) $\rho(\alpha x+\beta y) \leq \rho(x)+\rho(y)$ if and only if $\alpha+\beta=1$ and $\alpha, \beta \geq 0$,

(b) alternatively, if (3) is replaced by

(3)' $\rho(\alpha x+\beta y) \leq \alpha \rho(x)+\beta \rho(y)$ if and only if $\alpha+\beta=1$ and $\alpha, \beta \geq 0$,

then we say that $\rho$ is a convex modular.

A modular $\rho$ defines a corresponding modular space, i.e., the linear space $\chi_{\rho}$ given by

$$
\chi_{\rho}=\{x \in \chi: \rho(\lambda x) \rightarrow 0 \quad \text { as } \quad \lambda \rightarrow 0\} .
$$

Let $\rho$ be a convex modular. Then, the modular space $\chi_{\rho}$ can be equipped with a norm called the Luxemburg norm, defined by

$$
\|x\|_{\rho}=\inf \left\{\lambda>0: \rho\left(\frac{x}{\lambda}\right) \leq 1\right\} .
$$


A modular function $\rho$ is said to satisfy the $\Delta_{2}$-condition if there exists $\kappa>0$ such that $\rho(2 x) \leq \kappa \rho(x)$ for all $x \in \chi_{\rho}$.

Here, if the modular $\rho$ satisfies the $\Delta_{2}$-condition, we observe that $\kappa \geq 1$ for nontrivial modular $\rho$, and that $\kappa \geq 2$ for nontrivial convex modular $\rho$. Similarly, a modular function $\rho$ is said to satisfy the $\Delta_{3}$-condition if there exists $\kappa>0$ such that $\rho(3 x) \leq \kappa \rho(x)$ for all $x \in \chi_{\rho}$.

Remark 2. (a) If $\rho$ is a modular on $\chi$, we note that $\rho(t x)$ is an increasing function in $t \geq 0$ for each fixed $x \in \chi$, that is, $\rho(a x) \leq \rho(b x)$ whenever $0 \leq a<b$. (b) If $\rho$ is a convex modular on $\chi$ and $|\alpha| \leq 1$, then $\rho(\alpha x) \leq|\alpha| \rho(x)$ for all $x \in \chi$. Moreover, we see that $\rho(\alpha x) \leq \alpha \rho(x)$ for all $x \in \chi$, provided $0<\alpha \leq 1$, more generally, $\rho\left(\sum_{i=1}^{n} \alpha_{i} x_{i}\right) \leq \sum_{i=1}^{n} \alpha_{i} \rho\left(x_{i}\right)$ for all $x_{i} \in \chi$ and $\alpha_{i} \geq 0(i=1, \cdots, n)$ whenever $0<\sum_{i=1}^{n} \alpha_{i}:=\alpha \leq 1$. In fact, since $\sum_{i=1}^{n}\left(\frac{\alpha_{i}}{\alpha}\right)=1$,

$$
\begin{aligned}
\rho\left(\sum_{i=1}^{n} \alpha_{i} x_{i}\right) & =\rho\left(\alpha\left(\sum_{i=1}^{n} \frac{\alpha_{i}}{\alpha} x_{i}\right)\right) \\
& \leq \alpha \rho\left(\sum_{i=1}^{n} \frac{\alpha_{i}}{\alpha} x_{i}\right) \leq \alpha \sum_{i=1}^{n} \frac{\alpha_{i}}{\alpha} \rho\left(x_{i}\right)=\sum_{i=1}^{n} \alpha_{i} \rho\left(x_{i}\right)
\end{aligned}
$$

for all $x_{i} \in \chi$.

Definition 3. Let $\chi_{\rho}$ be a modular space and let $\left\{x_{n}\right\}$ be a sequence in $\chi_{\rho}$. Then,

(1) $\left\{x_{n}\right\}$ is $\rho$-convergent to $x \in \chi_{\rho}$ and write $x_{n} \stackrel{\rho}{\rightarrow} x$ if $\rho\left(x_{n}-x\right) \rightarrow 0$ as $n \rightarrow \infty$.

(2) $\left\{x_{n}\right\}$ is called $\rho$-Cauchy in $\chi_{\rho}$ if $\rho\left(x_{n}-x_{m}\right) \rightarrow 0$ as $n, m \rightarrow \infty$.

(3) A subset $K$ of $\chi_{\rho}$ is called $\rho$-complete if and only if any $\rho$-Cauchy sequence is $\rho$-convergent to an element in $K$.

They say that the modular $\rho$ has the Fatou property if and only if $\rho(x) \leq$ $\liminf _{n \rightarrow \infty} \rho\left(x_{n}\right)$ whenever the sequence $\left\{x_{n}\right\}$ is $\rho$-convergent to $x$.

Concerning the stability of functional equations, G. Sadeghi [10] has proved generalized Hyers-Ulam stability via the fixed point method of a generalized Jensen functional equation $f(r x+s y)=r g(x)+s h(y)$ in convex modular spaces with the Fatou property satisfying the $\Delta_{2}$-condition with $0<\kappa \leq 2$. In the paper [13], the authors have presented the generalized Hyers-Ulam stability of quadratic functional equations via the extensive studies of fixed point 
theory in the framework of modular spaces whose modulars are convex, lower semicontinuous but do not satisfy any relatives of $\Delta_{2}$-conditions. Recently, A. Zivari-Kazempour and M. Eshaghi Gordji [14] have determined the general solution of the quadratic functional equation

$$
\begin{aligned}
& f(x+2 y)+f(y+2 z)+f(z+2 x) \\
& \quad=2 f(x+y+z)+3[f(x)+f(y)+f(z)],
\end{aligned}
$$

which is equivalent to the quadratic functional equation

$$
f(x+y)+f(x-y)=2[f(x)+f(y)]
$$

and then they have proved its generalized Hyers-Ulam stability in normed spaces. In this article, we first present generalized Hyers-Ulam stability via direct method of the equation (1) in modular spaces without using the Fatou property and $\Delta_{3}$-conditions, and then investigate alternatively generalized Hyers-Ulam stability via direct method of the equation (1) in modular spaces using necessarily $\Delta_{3}$-conditions without the Fatou property.

\section{Generalized Hyers-Ulam Stability of Equation (1)}

Before making up the main subject, we use the following abbreviation for notational convenience :

$$
\begin{aligned}
D f(x, y, z):=\quad & f(x+2 y)+f(y+2 z)+f(z+2 x) \\
& -2 f(x+y+z)-3[f(x)+f(y)+f(z)]
\end{aligned}
$$

for all $x, y, z$ in a linear space $X$. In the following theorem, we first present a generalized Hyers-Ulam stability via direct method of the equation (1) in modular spaces without using the Fatou property and $\Delta_{3}$-condition.

Theorem 4. Let $X$ be a linear space and $\chi_{\rho}$ a $\rho$-complete convex modular space. Suppose that a mapping $f: X \rightarrow \chi_{\rho}$ satisfies

$$
\rho(D f(x, y, z)) \leq \phi(x, y, z)
$$

and $\phi: X^{3} \rightarrow[0, \infty)$ is a mapping such that

$$
\Phi(x, y, z):=\sum_{j=0}^{\infty} \frac{\phi\left(3^{j} x, 3^{j} y, 3^{j} z\right)}{9^{j}}<\infty
$$


for all $x, y, z \in X$. Then there exists a unique quadratic mapping $F_{1}: X \rightarrow \chi_{\rho}$ defined by $F_{1}(x):=\lim _{n \rightarrow \infty} \frac{f\left(3^{n} x\right)}{9^{n}}, x \in X$, which satisfies the equation (1) and

$$
\rho\left(f(x)-F_{1}(x)\right) \leq \frac{1}{9} \Phi(x, x, x)
$$

for all $x \in X$.

Proof. Letting $y=z:=x$ in (2), we obtain

$$
\rho(D f(x, x, x))=\rho(f(3 x)-9 f(x)) \leq \phi(x, x, x),
$$

and so

$$
\rho\left(f(x)-\frac{f(3 x)}{9}\right) \leq \frac{1}{9} \rho(f(3 x)-9 f(x)) \leq \frac{1}{9} \phi(x, x, x)
$$

for all $x \in X$. By induction on $n$, one can prove the following functional inequality

$$
\rho\left(f(x)-\frac{f\left(3^{n} x\right)}{9^{n}}\right) \leq \sum_{j=0}^{n-1} \frac{\phi\left(3^{j} x, 3^{j} x, 3^{j} x\right)}{9^{j+1}}
$$

for all $x \in X$.

Now, replacing $x$ by $3^{m} x$ in (6), we have

$$
\rho\left(\frac{f\left(3^{m} x\right)}{9^{m}}-\frac{f\left(3^{n+m} x\right)}{9^{n+m}}\right) \leq \frac{1}{9} \sum_{j=m}^{n+m-1} \frac{\phi\left(3^{j} x, 3^{j} x, 3^{j} x\right)}{9^{j}}
$$

which converges to zero as $m \rightarrow \infty$ by the assumption (3). Thus the above inequality implies that the sequence $\left\{\frac{f\left(3^{n} x\right)}{9^{n}}\right\}$ is $\rho$-Cauchy for all $x \in X$ and so it is convergent in $\chi_{\rho}$ since the space $\chi_{\rho}$ is $\rho$-complete. Thus, we may define a mapping $F_{1}: X \rightarrow \chi_{\rho}$ as

$$
F_{1}(x):=\rho-\lim _{n \rightarrow \infty} \frac{f\left(3^{n} x\right)}{9^{n}}, \quad(x \in X),
$$

which implies

$$
\lim _{n \rightarrow \infty} \rho\left(\frac{f\left(3^{n} x\right)}{9^{n}}-F_{1}(x)\right)=0, \quad(x \in X) .
$$

Now, we claim the mapping $F_{1}$ satisfies the equation (1). Setting $(x, y, z)$ $:=\left(3^{n} x, 3^{n} y, 3^{n} z\right)$ in $(2)$, and dividing the resulting inequality by $9^{n}$, we get

$$
\rho\left(\frac{D f\left(3^{n} x, 3^{n} y, 3^{n} z\right)}{9^{n}}\right) \leq \frac{\rho\left(D f\left(3^{n} x, 3^{n} y, 3^{n} z\right)\right)}{9^{n}} \leq \frac{\phi\left(3^{n} x, 3^{n} y, 3^{n} z\right)}{9^{n}}
$$


for all $x, y, z \in X$. Thus, it follows from the property $\rho(\alpha u) \leq \alpha \rho(u),(0<\alpha \leq$ $\left.1, u \in \chi_{\rho}\right)$ that

$$
\begin{aligned}
& \rho\left(\frac{1}{15} D F_{1}(x, y, z)\right) \\
& \leq \rho\left(\frac{1}{15} D F_{1}(x, y, z)-\frac{D f\left(3^{n} x, 3^{n} y, 3^{n} z\right)}{15 \cdot 9^{n}}+\frac{D f\left(3^{n} x, 3^{n} y, 3^{n} z\right)}{15 \cdot 9^{n}}\right) \\
& \leq \frac{1}{15} \rho\left(F_{1}(x+2 y)-\frac{f\left(3^{n}(x+2 y)\right)}{9^{n}}\right)+\frac{1}{15} \rho\left(F_{1}(y+2 z)-\frac{f\left(3^{n}(y+2 z)\right)}{9^{n}}\right) \\
& +\frac{1}{15} \rho\left(F_{1}(z+2 x)-\frac{f\left(3^{n}(z+2 x)\right)}{9^{n}}\right)+\frac{2}{15} \rho\left(F_{1}(x+y+z)-\frac{f\left(3^{n}(x+y+z)\right)}{9^{n}}\right) \\
& +\frac{3}{15} \rho\left(F_{1}(x)-\frac{f\left(3^{n} x\right)}{3^{n}}\right)+\frac{3}{15} \rho\left(F_{1}(y)-\frac{f\left(3^{n} y\right)}{\left.3^{n}\right)+\frac{3}{15} \rho\left(3_{1}(z)-\frac{f\left(3^{n}\right)}{3^{n}}\right)}\right. \\
& +\frac{1}{15} \rho\left(\frac{D f\left(3^{n} x, 3^{n} y, 3^{n} z\right)}{9^{n}}\right)
\end{aligned}
$$

for all $x, y, z \in X$ and all positive integers $n$. Taking the limit as $n \rightarrow \infty$, one obtains $\rho\left(\frac{1}{15} D F_{1}(x, y, z)\right)=0$, and so $D F_{1}(x, y, z)=0$ for all $x, y, z \in X$. Hence $F_{1}$ satisfies the equation (1) and so it is quadratic.

On the other hand, since $\sum_{i=0}^{n} \frac{1}{9^{i+1}}+\frac{1}{9} \leq 1$ for all $n \in \mathbb{N}$, it follows from (5) and the convexity of the modular $\rho$ that

$$
\begin{aligned}
& \rho\left(f(x)-F_{1}(x)\right) \\
& =\rho\left(-\sum_{i=0}^{n} \frac{1}{9^{i+1}} D f\left(3^{i} x, 3^{i} x, 3^{i} x\right)+\frac{f\left(3^{n+1} x\right)}{9^{n+1}}-\frac{F_{1}(3 x)}{9}\right) \\
& \leq \sum_{i=0}^{n} \frac{1}{9^{i+1}} \rho\left(D f\left(3^{i} x, 3^{i} x, 3^{i} x\right)\right)+\frac{1}{9} \rho\left(\frac{f\left(3^{n} \cdot 3 x\right)}{9^{n}}-F_{1}(3 x)\right) \\
& \leq \sum_{i=0}^{n} \frac{1}{9^{i+1}} \phi\left(3^{i} x, 3^{i} x, 3^{i} x\right)+\frac{1}{9} \rho\left(\frac{f\left(3^{n} \cdot 3 x\right)}{9^{n}}-F_{1}(3 x)\right),
\end{aligned}
$$

without applying the Fatou property of the modular $\rho$ for all $x \in X$ and all $n \in \mathbb{N}$, from which we obtain the approximation of $f$ by the quadratic mapping $F_{1}$ as follows

$$
\rho\left(f(x)-F_{1}(x)\right) \leq \sum_{i=0}^{\infty} \frac{1}{9^{i+1}} \phi\left(3^{i} x, 3^{i} x, 3^{i} x\right)=\frac{1}{9} \Phi(x, x, x)
$$

for all $x \in X$ by taking $n \rightarrow \infty$ in the last inequality. 
To show the uniqueness of $F_{1}$, we assume that there exists a quadratic mapping $G_{1}: X \rightarrow \chi_{\rho}$ which satisfies the inequality

$$
\rho\left(f(x)-G_{1}(x)\right) \leq \sum_{j=0}^{\infty} \frac{\phi\left(3^{j} x, 3^{j} x, 3^{j} x\right)}{9^{j+1}}
$$

for all $x \in X$, but suppose $F_{1}\left(x_{0}\right) \neq G_{1}\left(x_{0}\right)$ for some $x_{0} \in X$. Then there exists a positive constant $\varepsilon>0$ such that $\varepsilon<\rho\left(F_{1}\left(x_{0}\right)-G_{1}\left(x_{0}\right)\right)$. For such given $\varepsilon>0$, it follows from (3) that there is a positive integer $n_{0} \in \mathbb{N}$ such that $\sum_{j=n_{0}}^{\infty} \frac{\phi\left(3^{j} x_{0}, 3^{j} x_{0}, 3^{j} x_{0}\right)}{9^{j+1}}<\frac{\varepsilon}{2}$. Since $F_{1}$ and $G_{1}$ are quadratic mappings, we see from the equality $F_{1}\left(3^{n_{0}} x_{0}\right)=9^{n_{0}} F_{1}\left(x_{0}\right)$ and $G_{1}\left(3^{n_{0}} x_{0}\right)=9^{n_{0}} G_{1}\left(x_{0}\right)$ that

$$
\begin{aligned}
\varepsilon & <\rho\left(F_{1}\left(x_{0}\right)-G_{1}\left(x_{0}\right)\right) \\
& =\rho\left(\frac{F_{1}\left(3^{n_{0}} x_{0}\right)-f\left(3^{n_{0}} x_{0}\right)}{9^{n_{0}}}+\frac{f\left(3^{n_{0}} x_{0}\right)-G_{1}\left(3^{n_{0}} x_{0}\right)}{9^{n_{0}}}\right) \\
& \leq \frac{1}{9^{n_{0}}} \rho\left(F_{1}\left(3^{n_{0}} x_{0}\right)-f\left(3^{n_{0}} x_{0}\right)\right)+\frac{1}{9^{n_{0}}} \rho\left(f\left(3^{n_{0}} x_{0}\right)-G_{1}\left(3^{n_{0}} x_{0}\right)\right) \\
& \leq \frac{2}{9^{n_{0}}} \sum_{j=0}^{\infty} \frac{\phi\left(3^{j+n_{0}} x_{0}, 3^{j+n_{0}} x_{0}, 3^{j+n_{0}} x_{0}\right)}{9^{j+1}} \\
& \leq 2 \sum_{j=n_{0}}^{\infty} \frac{\phi\left(3^{j} x_{0}, 3^{j} x_{0}, 3^{j} x_{0}\right)}{9^{j+1}}<\varepsilon,
\end{aligned}
$$

which leads a contradiction. Hence the mapping $F_{1}$ is a unique quadratic mapping near $f$ satisfying the approximation (4) in the modular space $\chi_{\rho}$.

As corollaries of Theorem 4, we obtain the following stability results of the equation (1), which generalize stability results in normed spaces.

Corollary 5. Suppose $X$ is a linear space and $\chi_{\rho}$ is a $\rho$-complete convex modular space. Let $f: X \rightarrow \chi_{\rho}$ be a mapping satisfying

$$
\rho(D f(x, y, z)) \leq \varepsilon
$$

for some $\varepsilon>0$ and for all $x, y, z \in X$. Then there exists a unique quadratic mapping $F_{1}: X \rightarrow \chi_{\rho}$ which satisfies (1) and

$$
\rho\left(f(x)-F_{1}(x)\right) \leq \frac{\varepsilon}{8} \quad(x \in X)
$$


Corollary 6. Suppose $X$ is a normed space and $\chi_{\rho}$ is a $\rho$-complete convex modular space. For given real numbers $\varepsilon_{i}>0$ and $r_{i}<2(i=1,2,3)$, if a mapping $f: X \rightarrow \chi_{\rho}$ satisfies

$$
\rho(D f(x, y, z)) \leq \varepsilon_{1}\|x\|^{r_{1}}+\varepsilon_{2}\|y\|^{r_{2}}+\varepsilon_{3}\|z\|^{r_{3}}
$$

for all $x, y, z \in X$, then there exists a unique quadratic mapping $F_{1}: X \rightarrow \chi_{\rho}$ such that

$$
\rho\left(f(x)-F_{1}(x)\right) \leq \frac{\varepsilon_{1}\|x\|^{r_{1}}}{3^{2}-3^{r_{1}}}+\frac{\varepsilon_{2}\|x\|^{r_{2}}}{3^{2}-3^{r_{2}}}+\frac{\varepsilon_{3}\|x\|^{r_{3}}}{3^{2}-3^{r_{3}}}
$$

for all $x \in X$, where $x \neq 0$, if $r_{i}<0$.

Corollary 7. Suppose $X$ is a normed space and $\chi_{\rho}$ is a $\rho$-complete convex modular space. For given real numbers $\theta>0$ and $r_{i}(i=1,2,3)$, with $r<$ $2, r:=r_{1}+r_{2}+r_{3}$, if a mapping $f: X \rightarrow \chi_{\rho}$ satisfies

$$
\rho(D f(x, y, z)) \leq \theta\|x\|^{r_{1}}\|y\|^{r_{2}}\|z\|^{r_{3}}
$$

for all $x, y, z \in X$, then there exists a unique quadratic mapping $F_{1}: X \rightarrow \chi_{\rho}$ such that

$$
\rho\left(f(x)-F_{1}(x)\right) \leq \frac{\theta}{3^{2}-3^{r}}\|x\|^{r}, \quad(x \in X),
$$

where $x \neq 0$, if $r<0$.

We remark that a modular function $\rho$ satisfies the $\Delta_{3}$-condition if there exists $\kappa>0$ such that $\rho(3 x) \leq \kappa \rho(x)$ for all $x \in \chi_{\rho}$, where we note $\kappa \geq 3$ for nontrivial convex modular $\rho$ with $\Delta_{3}$-condition. So, we apply $\rho(9 x) \leq M \rho(x)$ for all $x \in \chi_{\rho}$, where $M:=\kappa^{2} \geq 9$ to the following theorem.

Theorem 8. Suppose $X$ is a linear space and $\chi_{\rho}$ is a $\rho$-complete convex modular space with $\Delta_{3}$-condition. If there exists a function $\varphi: X^{3} \rightarrow[0, \infty)$ for which a mapping $f: X \rightarrow \chi_{\rho}$ satisfies

$$
\begin{aligned}
\rho(D f(x, y, z)) & \leq \varphi(x, y, z), \quad \text { and } \\
\sum_{j=1}^{\infty} \frac{M^{2 j}}{9^{j}} \varphi\left(\frac{x}{3^{j}}, \frac{y}{3^{j}}, \frac{z}{3^{j}}\right) & :=\Psi(x, y, z)<\infty
\end{aligned}
$$

for all $x, y, z \in X$, then there exists a unique quadratic mapping $F_{2}: X \rightarrow \chi_{\rho}$, defined as $F_{2}(x)=\lim _{n \rightarrow \infty} 9^{n} f\left(\frac{x}{3^{n}}\right), x \in X$, which satisfies the equation (1) and

$$
\rho\left(f(x)-F_{2}(x)\right) \leq \frac{1}{9} \Psi(x, x, x)
$$

for all $x \in X$. 
Proof. We see from (5) that

$$
\rho\left(f(x)-9 f\left(\frac{x}{3}\right)\right) \leq \varphi\left(\frac{x}{3}, \frac{x}{3}, \frac{x}{3}\right)
$$

for all $x \in X$. Thus, by the convexity of the modular $\rho$ one has

$$
\begin{aligned}
\rho\left(f(x)-9^{2} f\left(\frac{x}{3^{2}}\right)\right) & \leq \frac{1}{9} \rho\left(9 f(x)-9^{2} f\left(\frac{x}{3}\right)\right)+\frac{1}{9} \rho\left(9^{2} f\left(\frac{x}{3}\right)-9^{3} f\left(\frac{x}{3^{2}}\right)\right) \\
& \leq \frac{M}{9} \varphi\left(\frac{x}{3}, \frac{x}{3}, \frac{x}{3}\right)+\frac{M^{2}}{9} \varphi\left(\frac{x}{3^{2}}, \frac{x}{3^{2}}, \frac{x}{3^{2}}\right)
\end{aligned}
$$

for all $x \in X$. Then it follows by induction on $n>1$ that

$$
\begin{aligned}
& \rho\left(f(x)-9^{n} f\left(\frac{x}{3^{n}}\right)\right) \\
& \quad \leq \sum_{j=1}^{n-1} \frac{M^{2 j-1}}{9^{j}} \varphi\left(\frac{x}{3^{j}}, \frac{x}{3^{j}}, \frac{x}{3^{j}}\right)+\frac{M^{2(n-1)}}{9^{n-1}} \varphi\left(\frac{x}{3^{n}}, \frac{x}{3^{n}}, \frac{x}{3^{n}}\right)
\end{aligned}
$$

for all $x \in X$. In fact, it is true for $n=2$. Assume that the inequality (12) holds true for $n$. Thus, using the convexity of the modular $\rho$, we deduce

$$
\begin{aligned}
& \rho\left(f(x)-9^{n+1} f\left(\frac{x}{3^{n+1}}\right)\right) \\
& =\rho\left(\frac{1}{9}\left(9 f(x)-9^{2} f\left(\frac{x}{3}\right)\right)+\frac{1}{9}\left(9^{2} f\left(\frac{x}{3}\right)-9^{n+2} f\left(\frac{x}{3^{n+1}}\right)\right)\right) \\
& \leq \frac{M}{9} \rho\left(f(x)-9 f\left(\frac{x}{3}\right)\right)+\frac{M^{2}}{9} \rho\left(f\left(\frac{x}{3}\right)-9^{n} f\left(\frac{x}{3^{n+1}}\right)\right) \\
& \leq \frac{M}{9} \varphi\left(\frac{x}{3}, \frac{x}{3}, \frac{x}{3}\right)+\frac{M^{2}}{9} \sum_{j=1}^{n-1} \frac{M^{2 j-1}}{9^{j}} \varphi\left(\frac{x}{3^{j+1}}, \frac{x}{3^{j+1}}, \frac{x}{3^{j+1}}\right) \\
& \quad+\frac{M^{2}}{9} \frac{M^{2(n-1)}}{9^{n-1}} \varphi\left(\frac{x}{3^{n+1}}, \frac{x}{3^{n+1}}, \frac{x}{3^{n+1}}\right) \\
& =\sum_{j=1}^{n} \frac{M^{2 j-1}}{9^{j}} \varphi\left(\frac{x}{3^{j}}, \frac{x}{3^{j}}, \frac{x}{3^{j}}\right)+\frac{M^{2 n}}{9^{n}} \varphi\left(\frac{x}{3^{n+1}}, \frac{x}{3^{n+1}}, \frac{x}{3^{n+1}}\right),
\end{aligned}
$$

which proves (12) for $n+1$. Now, replacing $x$ by $3^{-m} x$ in (12), we have

$$
\begin{aligned}
& \rho\left(9^{m} f\left(\frac{x}{3^{m}}\right)-9^{n+m} f\left(\frac{x}{3^{n+m}}\right)\right) \\
& \leq M^{m} \rho\left(f\left(\frac{x}{3^{m}}\right)-9^{n} f\left(\frac{x}{3^{n+m}}\right)\right)
\end{aligned}
$$




$$
\begin{aligned}
& \leq M^{m} \sum_{j=1}^{n-1} \frac{M^{2 j-1}}{9^{j}} \varphi\left(\frac{x}{3^{j+m}}, \frac{x}{3^{j+m}}, \frac{x}{3^{j+m}}\right)+M^{m} \frac{M^{2(n-1)}}{9^{n-1}} \varphi\left(\frac{x}{3^{n+m}}, \frac{x}{3^{n+m}}, \frac{x}{3^{n+m}}\right) \\
& \leq \frac{9^{m}}{M^{m}} \sum_{j=m+1}^{n+m-1} \frac{M^{2 j-1}}{9^{j}} \varphi\left(\frac{x}{3^{j}}, \frac{x}{3^{j}}, \frac{x}{3^{j}}\right)+\frac{9^{m}}{M^{m}} \frac{M^{2(n+m-1)}}{9^{n+m-1}} \varphi\left(\frac{x}{3^{n+m}}, \frac{x}{3^{n+m}}, \frac{x}{3^{n+m}}\right)
\end{aligned}
$$

which converges to zero as $m \rightarrow \infty$ by the assumption (10). Thus, the sequence $\left\{9^{n} f\left(\frac{x}{3^{n}}\right)\right\}$ is $\rho$-Cauchy for all $x \in X$ and so it is $\rho$-convergent in $\chi_{\rho}$ since the space $\chi_{\rho}$ is $\rho$-complete. Thus, we may define a mapping $F_{2}: X \rightarrow \chi_{\rho}$ as

$$
F_{2}(x):=\rho-\lim _{n \rightarrow \infty} 9^{n} f\left(\frac{x}{3^{n}}\right), \quad(x \in X),
$$

which implies

$$
\lim _{n \rightarrow \infty} \rho\left(9^{n} f\left(\frac{x}{3^{n}}\right)-F_{2}(x)\right)=0, \quad(x \in X) .
$$

Thus, by the $\Delta_{3}$-condition without using the Fatou property, we can see the following inequality

$$
\begin{aligned}
\rho\left(f(x)-F_{2}(x)\right) \leq & \frac{1}{9} \rho\left(9 f(x)-9^{n+1} f\left(\frac{x}{3^{n}}\right)+9^{n+1} f\left(\frac{x}{3^{n}}\right)-9 F_{2}(x)\right) \\
\leq & \frac{M}{9} \rho\left(f(x)-9^{n} f\left(\frac{x}{3^{n}}\right)\right)+\frac{M}{9} \rho\left(9^{n} f\left(\frac{x}{3^{n}}\right)-F_{2}(x)\right) \\
\leq & \frac{M}{9} \sum_{j=1}^{n-1} \frac{M^{2 j-1}}{9^{j}} \varphi\left(\frac{x}{3^{j}}, \frac{x}{3^{j}}, \frac{x}{3^{j}}\right)+\frac{M}{9} \frac{M^{2(n-1)}}{9^{n-1}} \varphi\left(\frac{x}{3^{n}}, \frac{x}{3^{n}}, \frac{x}{3^{n}}\right) \\
& \quad+\frac{M}{9} \rho\left(9^{n} f\left(\frac{x}{3^{n}}\right)-F_{2}(x)\right), \quad(\forall n \in \mathbb{N}) \\
\leq & \frac{1}{9} \sum_{j=1}^{\infty} \frac{M^{2 j}}{9^{j}} \varphi\left(\frac{x}{3^{j}}, \frac{x}{3^{j}}, \frac{x}{3^{j}}\right)
\end{aligned}
$$

by taking $n \rightarrow \infty$. Now, we claim the mapping $F_{2}$ satisfies the equation (1). Setting $(x, y, z):=\left(3^{-n} x, 3^{-n} y, 3^{-n} z\right)$ in (9), and multiplying the resulting inequality by $9^{n}$, we get

$$
\begin{aligned}
\rho\left(9^{n} D f\left(3^{-n} x, 3^{-n} y, 3^{-n} z\right)\right) & \leq M^{n} \varphi\left(3^{-n} x, 3^{-n} y, 3^{-n} z\right) \\
& \leq \frac{M^{2 n}}{9^{n}} \varphi\left(3^{-n} x, 3^{-n} y, 3^{-n} z\right),
\end{aligned}
$$

which tends to zero as $n \rightarrow \infty$ for all $x, y, z \in X$. Thus, it follows from the property $\rho(\alpha u) \leq \alpha \rho(u),\left(0<\alpha \leq 1, u \in \chi_{\rho}\right)$ that

$$
\rho\left(\frac{1}{15} D F_{2}(x, y, z)\right)
$$




$$
\begin{aligned}
= & \rho\left(\frac{1}{15} D F_{2}(x, y, z)-\frac{1}{15} 9^{n} \operatorname{Df}\left(\frac{x}{3^{n}}, \frac{y}{3^{n}}, \frac{z}{3^{n}}\right)+\frac{1}{15} 9^{n} \operatorname{Df}\left(\frac{x}{3^{n}}, \frac{y}{3^{n}}, \frac{z}{3^{n}}\right)\right) \\
\leq & \frac{1}{15} \rho\left(F_{2}(x+2 y)-9^{n} f\left(\frac{x+2 y}{3^{n}}\right)\right)+\frac{1}{15} \rho\left(F_{2}(y+2 z)-9^{n} f\left(\frac{y+2 z}{3^{n}}\right)\right) \\
& +\frac{1}{15} \rho\left(F_{2}(z+2 x)-9^{n} f\left(\frac{z+2 x}{3^{n}}\right)\right)+\frac{2}{15} \rho\left(F_{2}(x+y+z)-9^{n} f\left(\frac{x+y+z}{3^{n}}\right)\right) \\
& +\frac{3}{15} \rho\left(F_{2}(x)-9^{n} f\left(\frac{x}{3^{n}}\right)\right)+\frac{3}{15} \rho\left(F_{2}(y)-9^{n} f\left(\frac{y}{3^{n}}\right)\right)+\frac{3}{15} \rho\left(F_{2}(z)-9^{n} f\left(\frac{z}{3^{n}}\right)\right) \\
& +\frac{1}{15} \rho\left(9^{n} \operatorname{Df}\left(\frac{x}{3^{n}}, \frac{y}{3^{n}}, \frac{z}{3^{n}}\right)\right)
\end{aligned}
$$

for all $x, y, z \in X$. Taking the limit as $n \rightarrow \infty$, one obtains

$$
\rho\left(\frac{1}{15} D F_{2}(x, y, z)\right)=0
$$

and thus $D F_{2}(x, y, z)=0$ for all $x, y, z \in X$. Hence $F_{2}$ satisfies the equation (1), and so it is quadratic.

To show the uniqueness of $F_{2}$, we assume that there exists a quadratic mapping $G_{2}: X \rightarrow \chi_{\rho}$ which satisfies the inequality

$$
\rho\left(f(x)-G_{2}(x)\right) \leq \frac{1}{9} \sum_{j=1}^{\infty} \frac{M^{2 j}}{9^{j}} \varphi\left(\frac{x}{3^{j}}, \frac{x}{3^{j}}, \frac{x}{3^{j}}\right)
$$

for all $x \in X$. Since $F_{2}$ and $G_{2}$ are quadratic mappings, we see from the equality $9^{n} F_{2}\left(3^{-n} x\right)=F_{2}(x)$ and $9^{n} G_{2}\left(3^{-n} x\right)=G_{2}(x)$ that

$$
\begin{aligned}
\rho\left(G_{2}(x)-F_{2}(x)\right) & =\rho\left(\frac{9^{n+1}}{9}\left(G_{2}\left(\frac{x}{3^{n}}\right)-f\left(\frac{x}{3^{n}}\right)\right)+\frac{9^{n+1}}{9}\left(f\left(\frac{x}{3^{n}}\right)-F_{2}\left(\frac{x}{3^{n}}\right)\right)\right) \\
& \leq \frac{M^{n+1}}{9} \rho\left(G_{2}\left(\frac{x}{3^{n}}\right)-f\left(\frac{x}{3^{n}}\right)\right)+\frac{M^{n+1}}{9} \rho\left(f\left(\frac{x}{3^{n}}\right)-F_{2}\left(\frac{x}{3^{n}}\right)\right) \\
& \leq \frac{2 M^{n+1}}{9^{2}} \sum_{j=1}^{\infty} \frac{M^{2 j}}{9^{j}} \varphi\left(\frac{x}{3^{j+n}}, \frac{x}{3^{j+n}}, \frac{x}{3^{j+n}}\right) \\
& \leq \frac{2 M}{9^{2}} \frac{9^{n}}{M^{n}} \sum_{j=1}^{\infty} \frac{M^{2(j+n)}}{9^{j+n}} \varphi\left(\frac{x}{3^{j+n}}, \frac{x}{3^{j+n}}, \frac{x}{3^{j+n}}\right)
\end{aligned}
$$

which tends to zero as $n \rightarrow \infty$ for all $x \in X$. Hence the mapping $F_{2}$ is a unique quadratic mapping satisfying (11).

Remark 9. In Theorem 8 , if $\chi_{\rho}$ is a Banach space with norm $\rho$, and so $\rho(3 x)=3 \rho(x), \kappa:=3$, then we see from (9) and (10) that there exists a unique 
quadratic mapping $F_{2}: X \rightarrow \chi_{\rho}$, defined as $F_{2}(x)=\lim _{n \rightarrow \infty} 9^{n} f\left(\frac{x}{3^{n}}\right), x \in X$, which satisfies the equation (1) and

$$
\rho\left(f(x)-F_{2}(x)\right) \leq \frac{1}{9} \sum_{j=1}^{\infty} 9^{j} \varphi\left(\frac{x}{3^{j}}, \frac{x}{3^{j}}, \frac{x}{3^{j}}\right)
$$

for all $x \in X$, which is an implicit alternative stability result in the paper [14].

As corollaries of Theorem 8, we obtain the following stability results of the equation (1), which generalize stability results in normed spaces.

Corollary 10. Suppose $X$ is a normed space and $\chi_{\rho}$ is a $\rho$-complete convex modular space with $\Delta_{3}$-condition. For given real numbers $\varepsilon_{i}>0$ and $r_{i}>0$ subject to $\kappa^{4}<3^{2+r_{i}}(i=1,2,3)$, if a mapping $f: X \rightarrow \chi_{\rho}$ satisfies

$$
\rho(D f(x, y, z)) \leq \varepsilon_{1}\|x\|^{r_{1}}+\varepsilon_{2}\|y\|^{r_{2}}+\varepsilon_{3}\|z\|^{r_{3}}
$$

for all $x, y, z \in X$, then there exists a unique quadratic mapping $F_{2}: X \rightarrow \chi_{\rho}$ such that

$$
\rho\left(f(x)-F_{2}(x)\right) \leq \frac{\kappa^{4} \varepsilon_{1}\|x\|^{r_{1}}}{9\left(3^{2+r_{1}}-\kappa^{4}\right)}+\frac{\kappa^{4} \varepsilon_{2}\|x\|^{r_{2}}}{9\left(3^{2+r_{2}}-\kappa^{4}\right)}+\frac{\kappa^{4} \varepsilon_{3}\|x\|^{r_{3}}}{9\left(3^{2+r_{3}}-\kappa^{4}\right)}
$$

for all $x \in X$.

Corollary 11. Suppose $X$ is a vector space and $\chi_{\rho}$ is a $\rho$-complete convex modular space with $\Delta_{3}$-condition. For given real numbers $\theta>0$ and $r_{i}>0(i=$ $1,2,3)$, subject to $\kappa^{4}<3^{2+r}$, where $r:=r_{1}+r_{2}+r_{3}$, if a mapping $f: X \rightarrow \chi_{\rho}$ satisfies

$$
\rho(D f(x, y, z)) \leq \theta\|x\|^{r_{1}}\|y\|^{r_{2}}\|z\|^{r_{3}}
$$

for all $x, y, z \in X$, then there exists a unique quadratic mapping $F_{2}: X \rightarrow \chi_{\rho}$ such that

$$
\rho(f(x)-F(x)) \leq \frac{\kappa^{4} \theta}{9\left(3^{2+r}-\kappa^{4}\right)}\|x\|^{r}
$$

for all $x \in X$.

Remark 12. In Corollary 11, if $f(0)=0$ and $r_{i}>0$ for some $i=1,2,3$, then we find that $\operatorname{Df}(x, y, 0)=0$ for the case $r_{3}>0$ without loss of generality, and so $f$ is itself quadratic in this case. 


\section{Acknowledgments}

This research was supported by Basic Science Research Program through the National Research Foundation of Korea(NRF) funded by the Ministry of Education(2016R1D1A3B03930971).

\section{References}

[1] Y. Benyamini and J. Lindenstrauss, Geometric Nonlinear Functional Analysis, Vol. 1, Colloq. Publ. Vol. 48, Amer. Math. Soc. Providence, 2000, doi: 10.1090/coll/048.

[2] C. Borelli and G.L. Forti, On a general Hyers-Ulam stability result, Internat. J. Math. Math. Sci., 18 (1995), 229-236, doi: 10.1155/S0161171295000287.

[3] S. Czerwik, On the stability of the quadratic mapping in normed spaces, Abh. Math. Sem. Univ. Hamburg, 62 (1992), 59-64, doi: 10.1007/BF02941618.

[4] P. Găvruta, A generalization of the Hyers-Ulam-Rassias stability of approximately additive mappings, J. Math. Anal. Appl. 184 (1994), 431-436, doi: 10.1006/jmaa.1994.1211.

[5] D.H. Hyers, On the stability of the linear functional equation, Proc. Nat. Acad. Sci., USA, 27 (1941), 222-224, doi: 10.1073/pnas.27.4.222.

[6] J.M. Rassias, On the stability of the Euler-Lagrange functional equation, Chinese J. Math., 20 (1992), No. 2, 185-190.

[7] J.M. Rassias and H. Kim, Generalized Hyers-Ulam stability for general additive functional equations in quasi- $\beta$-normed spaces, J. Math. Anal. Appl., 356 (2009), 302-309, doi: $10.1016 /$ j.jmaa.2009.03.005.

[8] Th.M. Rassias, On the stability of the linear mapping in Banach spaces, Proc. Amer. Math. Soc., 72 (1978), 297-300, doi: 10.1090/S0002-9939-1978-0507327-1.

[9] S. Rolewicz, Metric Linear Spaces, Reidel/PWN-Polish Sci. Publ. Dordrecht, 1984.

[10] G. Sadeghi, A fixed point approach to stability of functional equations in modular spaces, Bull. Malays. Math. Sci. Soc., 37, No. 2 (2014), 333-344.

[11] F. Skof, Local properties and approximations of operators, Rend. Sem. Mat. Fis. Milano, 53 (1983), 113-129.

[12] S.M. Ulam, Problems in Modern Mathematics, Chap. VI, Wiley, New York, (1960).

[13] K. Wongkum, P. Chaipunya, and P. Kumam, On the generalized Ulam-Hyers-Rassias stability of quadratic mappings in modular spaces without $\Delta_{2}$-conditions, J. Funct. Spaces, Vol. 2015, Article ID 461719, 6 pages, doi: 10.1155/2015/461719.

[14] A. Zivari-Kazempour and M. Eshaghi Gordji, Generalized Hyers-Ulam Stabilities of an Euler-Lagrange-Rassias quadratic functional equation, Asian European J. Math., 5 (2012), doi: 10.1142/S1793557112500143. 
